\title{
Design and Implementation of a Novel WAMPAC System for Power Network with Remote Monitoring and Control
}

\author{
R. S. RajPrasad \\ PG student,(M.E-Power Systems Engineering) \\ Department of Electrical \& Electronics Engineering \\ Velammal Engineering College \\ Chennai-600066, India
}

\begin{abstract}
The proposed module is an innovative version which is developed to prove as an effective alternative model to the existing modules of phasor measurement units (PMU), ensuring feasible and reliable operation at very low design cost. The intricate design and circuitry involved in the WAMPAC systems are replaced with a cost-effective equivalent module for distribution feeders. Remote monitoring and control of the system is effectively designed so as to reduce the involvement of manpower and ease the outlook of complexity in power system protection.
\end{abstract}

Keywords: Capacitive Voltage Transformer, Data Sampling Unit, Database Management System, Data Concentrator Unit, Global Positioning System, Human Machine Interface, Time-stamped Data Log.

\section{Introduction}

The evolution of Power System Protection, on a longer run has fueled the development and growth of the power sector, assuring reliability by ensuring effective response to all the "pros and cons', faced by the Power System. Nowadays, "Power System Protection" itself is seen as an art and science of detecting problems incurred in the power system and execute a relevant control action to mitigate the fault.

The recent extension of protection system is the evolution of Phasor Measurement Units (PMU), which has taken the starlight in the power industry, for its faster response to faults and the wider extension of protection. However, taking the cost into consideration, the installation cost incurred with the PMU's is a costly affair, that it is difficult for the lower economical sector to afford. Power System Security and faster response to system faults is considered as the important yardstick for development of Protection Systems. So, taking faster response characteristics and Cost-considerations in mind, an alternative module is designed and suggested in this paper, which will benefit all economical classes of the Power Sector.

\section{Overview of Proposed Module}

The proposed module comprises of Data Sampling Units, Data Concentrator Units, Master Controller Unit and User Friendly HMI (Human Machine Interface) Unit for enhancing real-time Monitoring and Control.

In this module, both wired as well as wireless mode of communication are employed. For local communication, wired Communication is deployed and for remote access, wireless communication platform is deployed. For the purpose of manipulating time stamped measurements, GPS (Global Positioning System) unit is interfaced with the module.

\section{Data Sampling Unit}

The Data Sampling Units are the primary circuits crafted so as to tap the feeder parameters and sample the data in such a way that it serves to be suitable for the next processing sections.

As shown in the block diagram, the sampling unit for one side of the feeder is represented. The input signals for the sampling unit are tapped from the panel terminal block of the feeder, where the Secondary of Capacitive Voltage Transformers (CVT) and the Secondary of the Current Transformers (CT) will be terminated. The voltages and frequencies are conditioned by the voltage and frequency sampling unit respectively. 


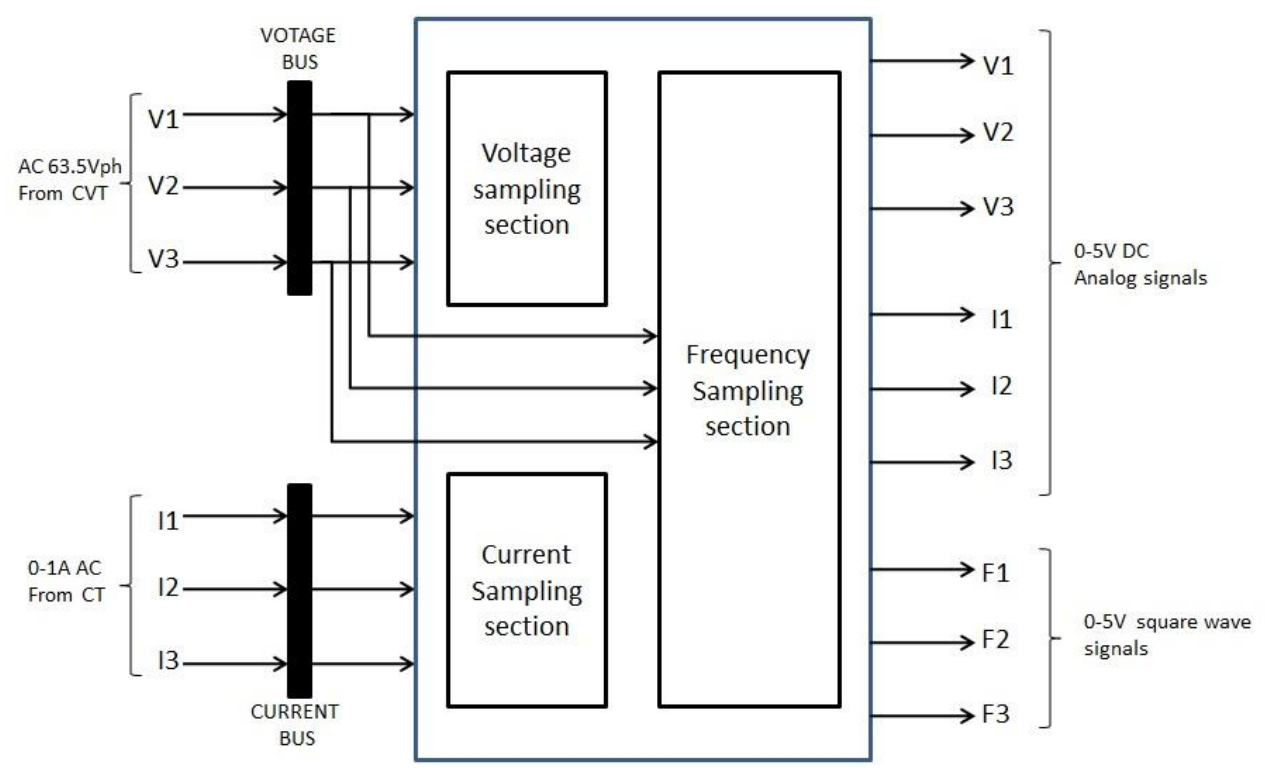

Fig. 1: Data Sampling Unit.

\subsection{Voltage Sampling Unit}

The Output of the primary sampling is $\mathrm{V}_{\mathrm{L}}$ or $63.5 \mathrm{~V}_{\mathrm{Ph}}$, which in turn is further sampled by a $230 / 0-12 \mathrm{~V}$ step down transformer. The Sampled AC Voltage is fed to an AC to DC converter, as the microcontroller unit has to be fed with $0-5 \mathrm{~V}$ DC signal.
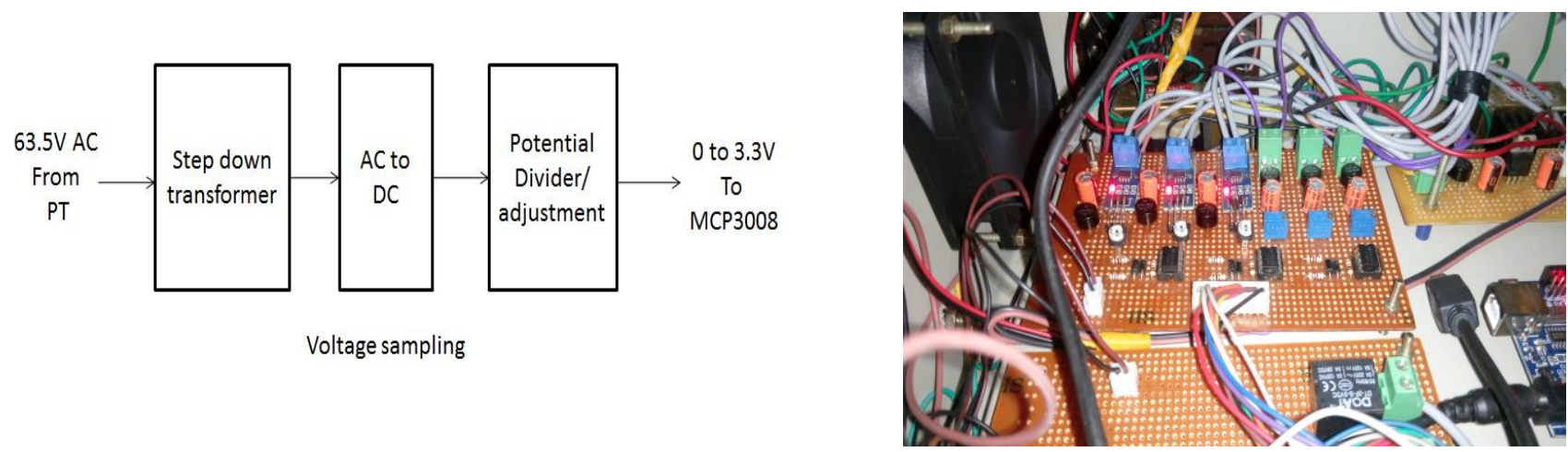

Fig. 2: Block Diagram and Hardware Circuitry of Voltage Sampling Unit.

The secondary of the CVT, which will be in the range of $0-63.5 \mathrm{~V}$, is stepped down to a range of $0-5 \mathrm{~V}$ AC. This $0-5 \mathrm{~V}$ $\mathrm{AC}$ is fed to a bridge rectifier, and a corresponding DC supply is generated so as to make the signal suitable for further processing in the controller. Output of full wave rectifier is not a constant DC voltage. It is observed from the output diagram that it is a pulsating DC voltage with AC ripples. In real life applications, a power supply with smooth wave form is required. In other words, a DC power supply with constant output voltage is desired. A constant output voltage from the DC power supply is very important as it directly impacts the reliability of the electronic device which is connected to the power supply. The output of full wave rectifier is made smoother by using a filter (a capacitor filter or an inductor filter) across the diode. In some cases, a resistor-capacitor coupled filter (RC) is also used.

\subsection{Current Sampling Unit}

The Output of the Primary sampling is $I_{L}=0-1 \mathrm{~A}$, which is fed to the input terminals of ACS712 Current sensor. The ACS712 outputs an analog signal, $V_{\text {OUT. }}$ which varies linearly with the unidirectional or bi-directional AC or DC sensed current from the primary, $\mathrm{I}_{\mathrm{P}}$, within the range specified. Capacitor Filter is recommended for noise management, with values that depend on the application. 

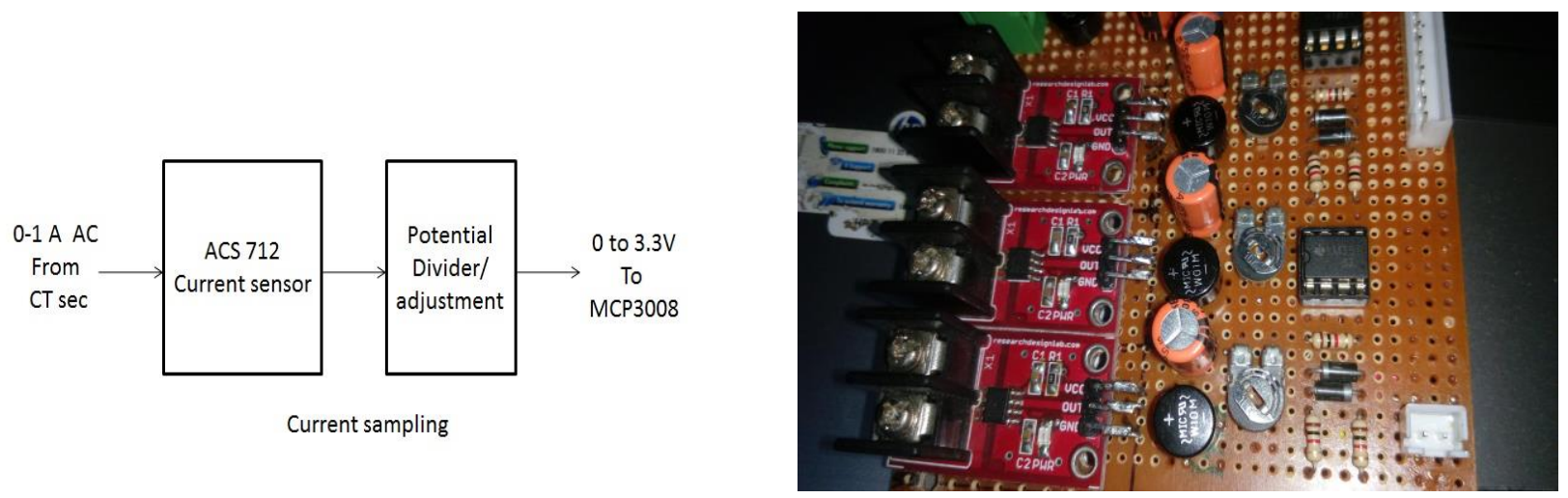

Fig. 3: Block Diagram and Hardware Circuitry of Current Sampling Unit.

The device consists of accurate, low-offset, linear Hall sensor circuit with a copper conduction path located near the surface of the Die. Applied current flowing through this copper conduction path generates a magnetic field which is sensed by the integrated Hall IC and converted into a proportional voltage. This device is a Hall Effect transducer. It should not be used near significant magnetic fields.

\subsection{Frequency Sampling Unit}

The Output of the Step down transformer is fed to an OP-AMP (LM358N) based zero-cross detector. The zero cross detector converts input $\mathrm{AC}$ sine wave into its corresponding square wave signal without changing its frequency. The Circuitry of the frequency sampling circuit is shown below.

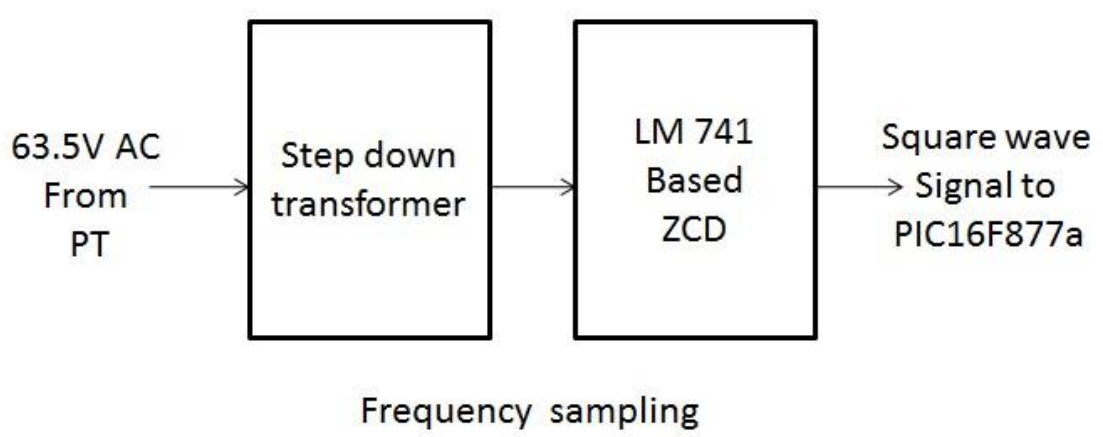

Fig. 4: Frequency Sampling Unit.

Zero crossing detector is used to convert sine wave or other signal into square-wave, the output should be low if the input is negative and high if the input if positive. A zero crossing detector literally detects the transition of a signal waveform from positive and negative, ideally providing a narrow pulse that coincides exactly with the zero voltage condition.

The diode D1 remains forward-biased, thereby allowing only the positive pulses and the diode D2, which remains reverse-biased, allows only the negative pulses. Thus, the diodes D1 and D2 doesn't allow intermittent pulses other than positive and negative pulses. Thus, based on the repetitive zero crossings, the frequency is analyzed.

\section{Data Concentrator Unit}

The Data Concentrator Unit is the terminal hub, where all the sampled signals from the sampling unit culminates. The Data Concentrator is interlinked with a GPS unit, so as to manipulate time-stamped measurements. The time-stamped measurements are measured at all nodal points wherever they are assigned to be measured. The Data Concentrator Unit, interfaced with GPS module, carries out time-stamped measurements and simultaneously records it in a database. The time of measurement along with the measured parameters are recorded continuously and communicated to the master controller. 


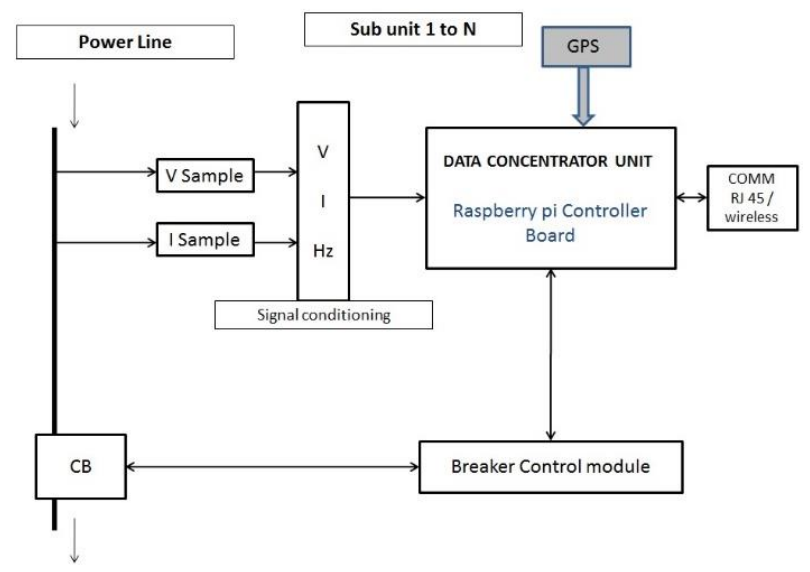

Fig. 5: Block Diagram of Data Concentrator Unit

The time-stamped measurements from the data concentrator are simultaneously communicated to the master controller at the remote end and the substation controller at the local end. For master controller, Wireless communication is deployed, while for Local controller, wired communication is deployed. Thus, a two stage protection scheme is developed, so as to create a module with $100 \%$ reliability.

\section{Master PDC}

The master PDC is located at the remote end and the main function of this unit is to establish communication between the Data Concentrator units and import time-stamped measurements from the units. The Master PDC is interfaced with the Data management system and all the time-stamped measurements are logged in the Database.

The Master PDC is responsible for establishing remote monitoring and control of the proposed module. The Communication Platform between the Data Concentrator Units and the Master PDC is Wireless as the Master PDC will be located at the remote end.

\section{Protection Schemes}

There are numerous Protection Schemes available for different zones of the power system. The Choice of the Protection Schemes to be employed in the module depends on the requisite of the User and the module can be customized based on the User requirements.

In this module, the Protection Schemes are focused on Feeder Protection. Although Feeder Protection involves a lot of Schemes, some major Protective Schemes are considered to develop this Prototype. The Protection Schemes inculcated in this Module are segregated to be programmed in master controller and Feeder side Controller depending on the data fetched, i.e, in the feeder side controller, the data from that particular feeder alone is fetched whereas in the master controller, data from all nodes are fetched. The Various Protection Schemes employed in the module are as follows.

1. Distance Protection Scheme

2. Overcurrent Protection Scheme

3. Switched On to Earth Fault Scheme

4. Under Voltage Protection Scheme

5. Over Voltage Protection scheme

6. Differential Protection Scheme

7. Check Synchronizing Protection Scheme

The Differential Protection Scheme and Check Synchronizing Protection schemes are programmed in the Master Controller, while the rest are programmed in the Feeder Side Controller and Substation Controller. These are the various Protection Schemes incorporated within the proposed module, though any kind of protection Scheme logic can be incorporated in the system. 


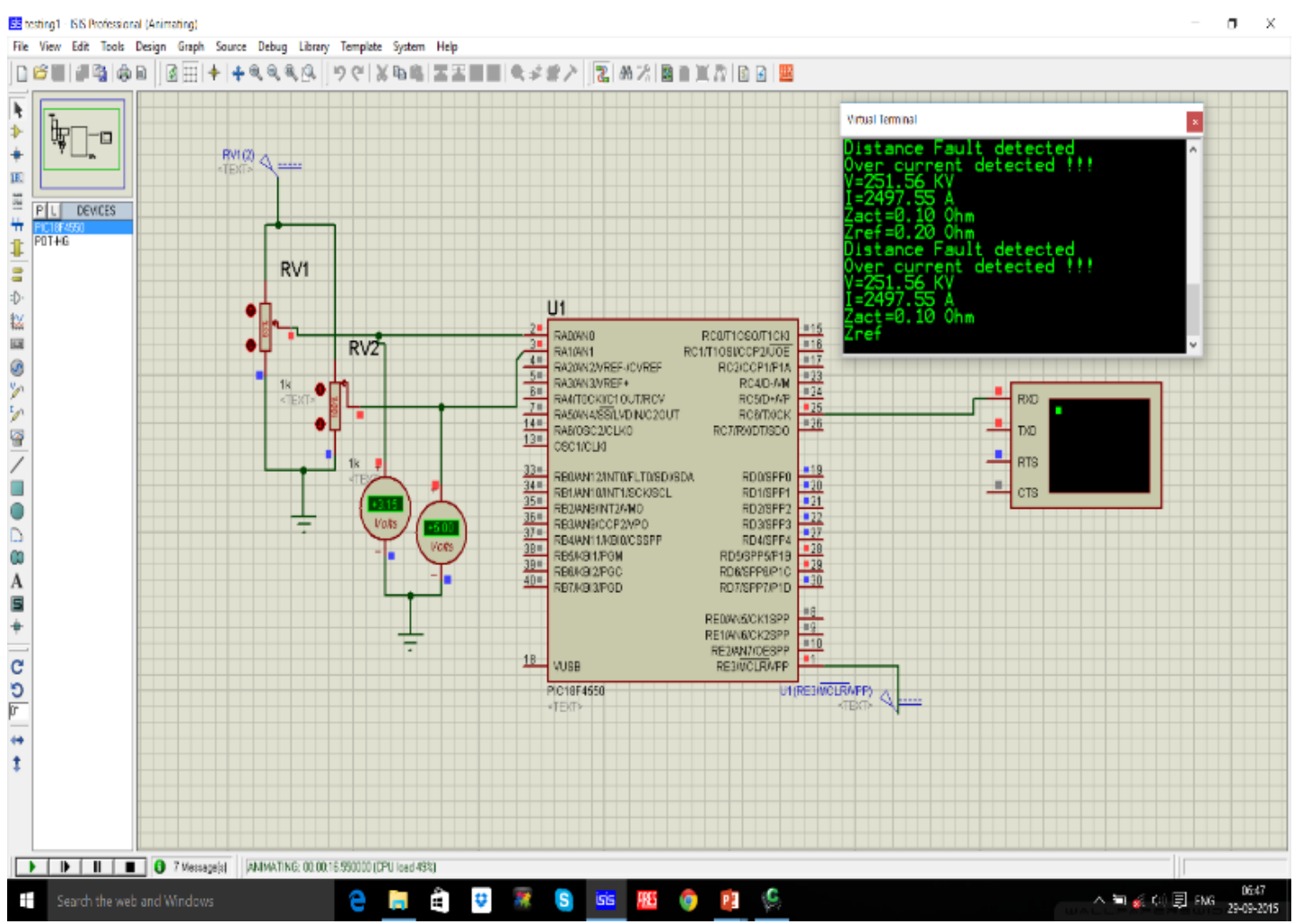

Fig. 6: Simulation of the Protection Schemes.

The Simulated results of the Protection Scheme logic is shown above. In the simulation, the cut-off limits of a typical $400 \mathrm{kV}$ feeder is considered as reference values. The controller is the integral part of the PDC unit and when it is interfaced with a GPS module, time-stamped measurements are fetched and manipulated. The PDC simultaneously communicates the fetched data and the corresponding control action to the Master controller and all other associated subsystems. The Master PDC, or the Remote Master System Controller (RMSC) collects all the data and logs it in a common database. It also performs the operation of executing the relay schemes like Check Synchronizing and Differential Protection Schemes, which has to be performed using the data collected from multiple feeder points. Based on the cumulative data collected and logged on time-stamped basis, a detailed and accurate analysis of the power network can be carried out. The sequence of events, to which the system is subjected to can easily be analyzed and the magnitude of fault being extended further is prevented, thereby ensuring system reliability. In addition to logging of measured data, the corrective actions taken for the corresponding event will also be recorded. These are the Various Protection Schemes imbibed within the proposed module. It has to be noted that, depending on the User requirements, the analysis to be carried out with the acquired data can be customized.

\section{Communication Protocol}

Communication is a vital segment in this module, as it is the nerve that helps in transmitting all the collected timestamped measurements between various nodal points in the network. To establish this effectively, the proposed communication strategy is designed to suit the need and purpose of this module and is executed accordingly.

The Data Concentrator at the Local end communicates the measured signals with the Router, which is established through Local Area Network (LAN). The Data Packets are transmitted to the Remote Master System Controller (RMSC), which is at the remote end and this is established through wireless communication through Wide Area network (WAN) Protocol.

An innovative trend of Monitoring and Control strategy entitled as "Anytime Anywhere Access Protocol" has been framed and developed wherein the System Performance can be monitored and controlled anywhere, provided the User or Engineer strictly abides by the terms and norms of Data Encryption and Data Security. Data Encryption and Data Security is a challenging task in visualizing this protocol as only officially designated Operators can log into the control module while others can just observe the system performance. 


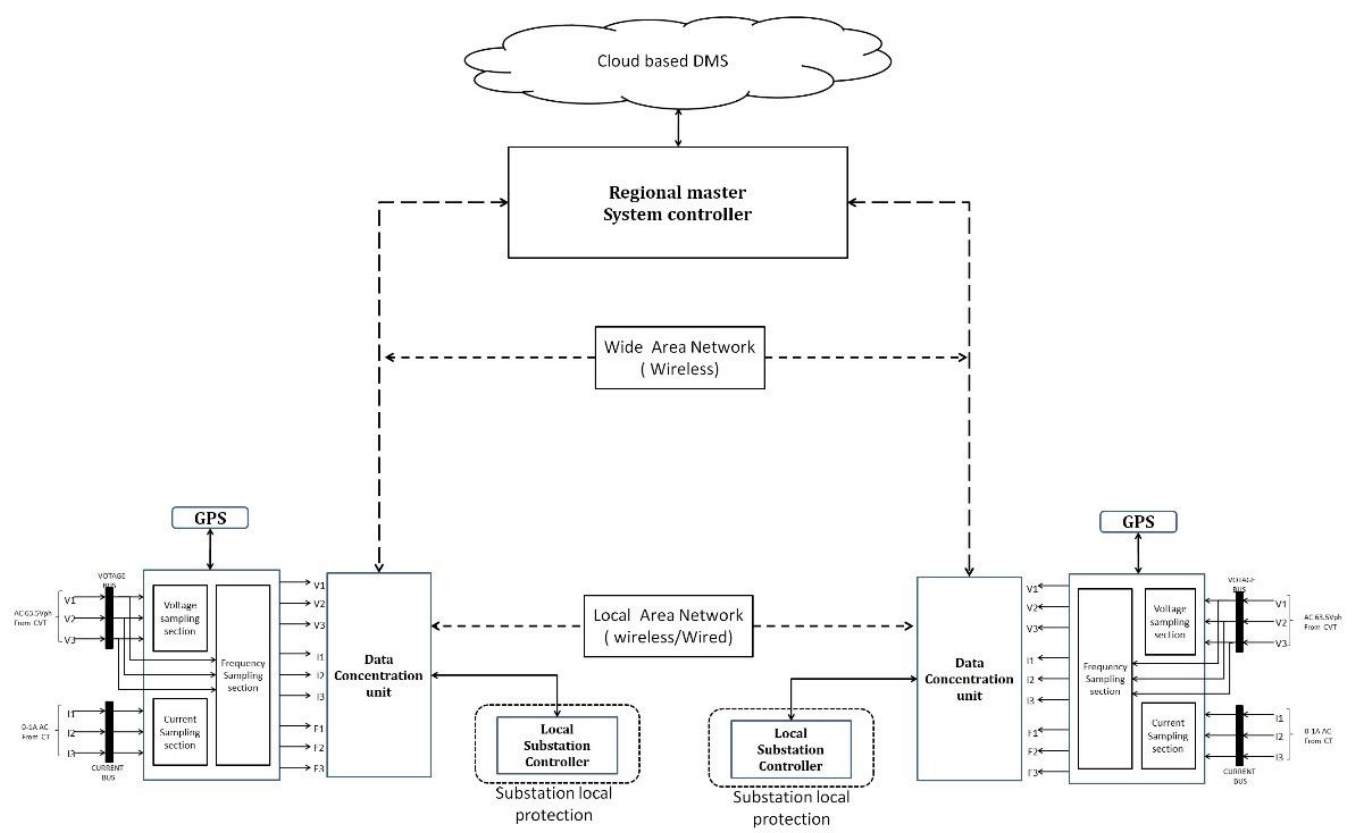

Fig. 7: Overall Block Diagram of the Proposed Module.

The Interesting concept in this proposed Module is that it is not necessary for the Engineer or Control Room Operator to always stay near the Panels and keep monitoring. The Engineer can freely move anywhere with all the system parameters in the tip of his hands and can easily control the system. This concept steers the existing power technology towards the revolution of Unmanned Power Stations.

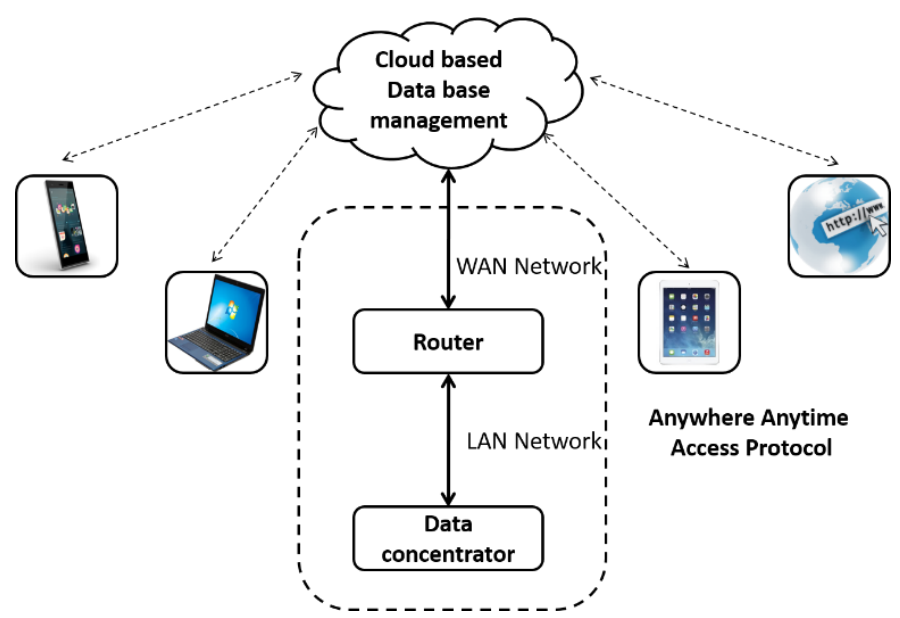

Fig. 8: Design of the adopted Communication method for the module.

\section{Graphical User Interface}

The Graphical User Interface, commonly termed as the GUI or SCADA (Supervisory Control and Data Acquisition System) is an Interface that allows the User or Control Engineer to monitor and control the System. To establish the GUI for this module, "LabVIEW" has been used as a development Platform. User Easiness and Reducing the complexity of Module has been given utmost importance while developing the Human Machine Interface.

The Casting of Data can be done in any customized form depending on the User requisite. The Crafting of the GUI involves designing the Front Panel and Back Panel. The Front Panel is the interface on which the user will be able to Monitor and Control. In the Back Panel, the interfacing logic between the hardware and the Front Panel is designed. 


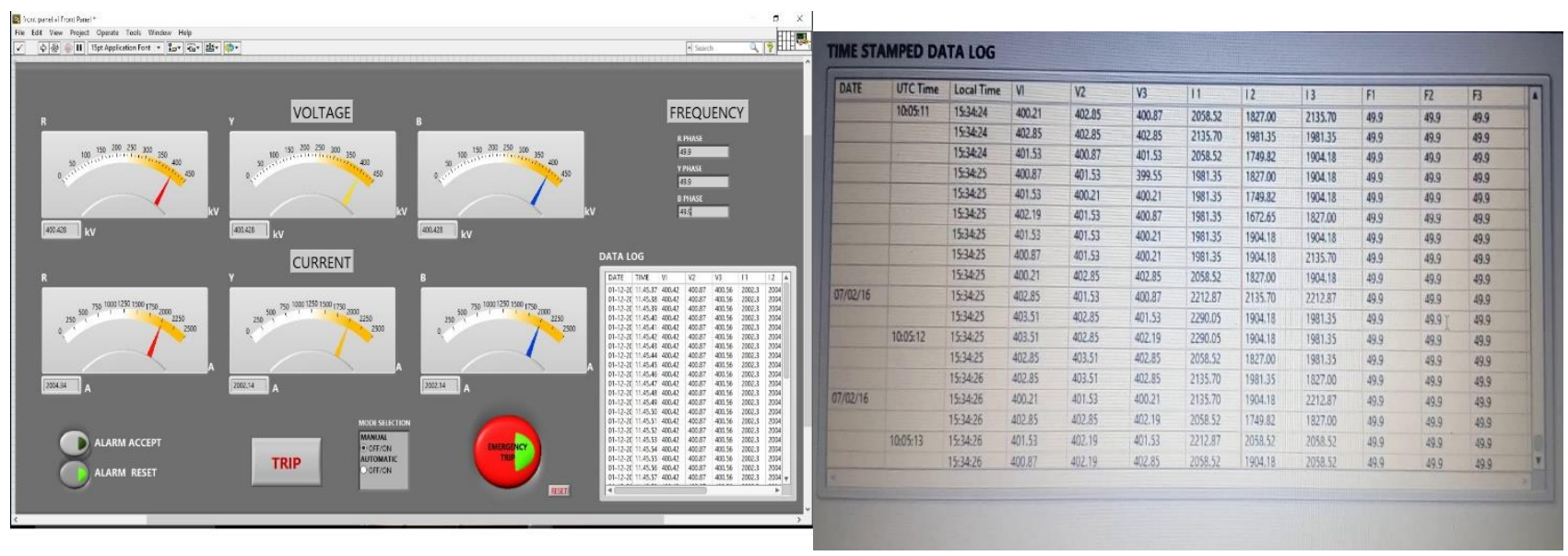

Fig. 9: Front panel of GUI with Time Stamped Measurements.

The Picture illustrated above represents the front panel of the GUI. The front panel is crafted with analog and digital meters to cast the measured voltage, current and frequency. The Control section in this GUI involves resetting of Alarm signals, Trip Button, Emergency Trip Button and mode selection for toggling between manual and automatic modes. Apart from these, "Synchronization" can also be done in the RMSC GUI. Time-stamped Data logging is also included to perform a detailed analysis of the sequence of events in the system. This kind of a User-Friendly Interface reduces the complexity of the entire Power System Operation and Control, thereby paving the way for Automated Power Stations.

\section{Hardware Conception of the Proposed Module}

The Conception of the Hardware Module for the Proposed Concept is picturized in the Snapshot shown below.

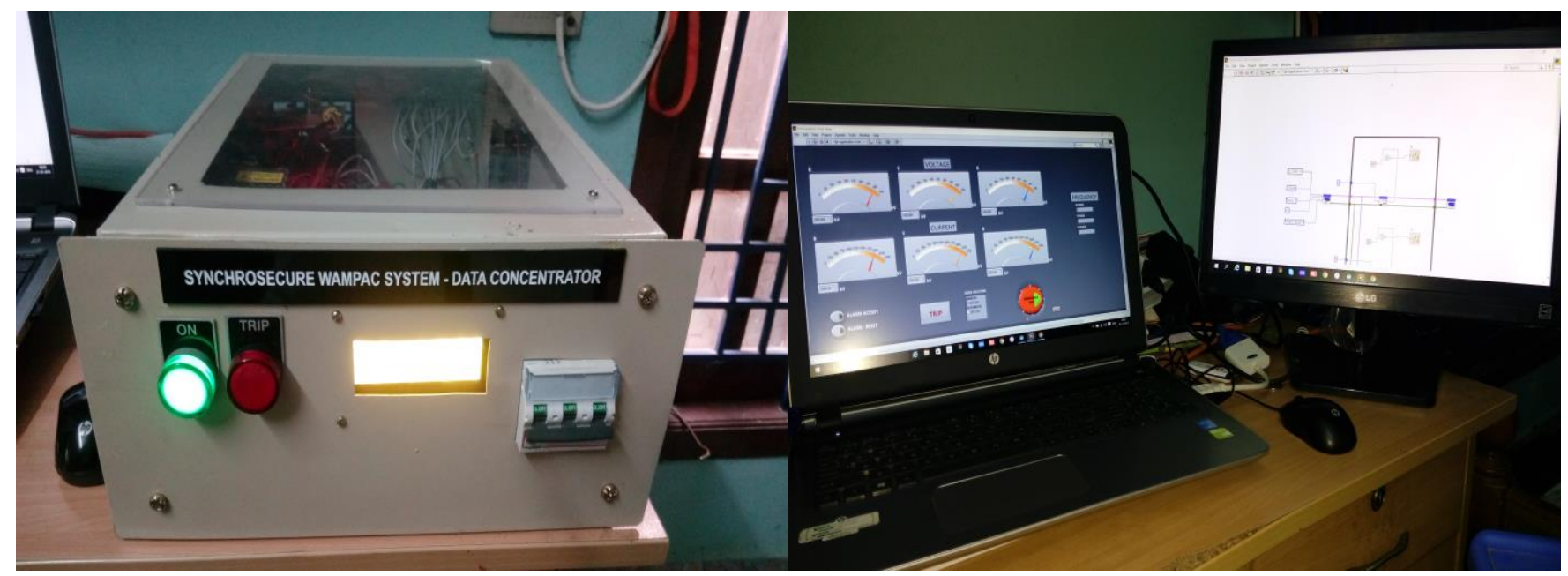

Fig. 10: Hardware Conception and the HMI Unit of the proposed Module.

The module has also been tested for its reliability by injecting the Voltage and Current Signals and triggering various kinds of faults to ensure that all the Protection Schemes work satisfactorily. Omicron CMC 256 plus has been used to inject the corresponding input Voltages and Currents during the testing process.

All the Control logics including the Trip Signal, Emergency Trip Signal, Annunciation Accept, Annunciation Reset were tested in both Manual and Automatic mode of Operation. The Pictorial representation shown below is the hardware setup of the designed module during the process of testing. 


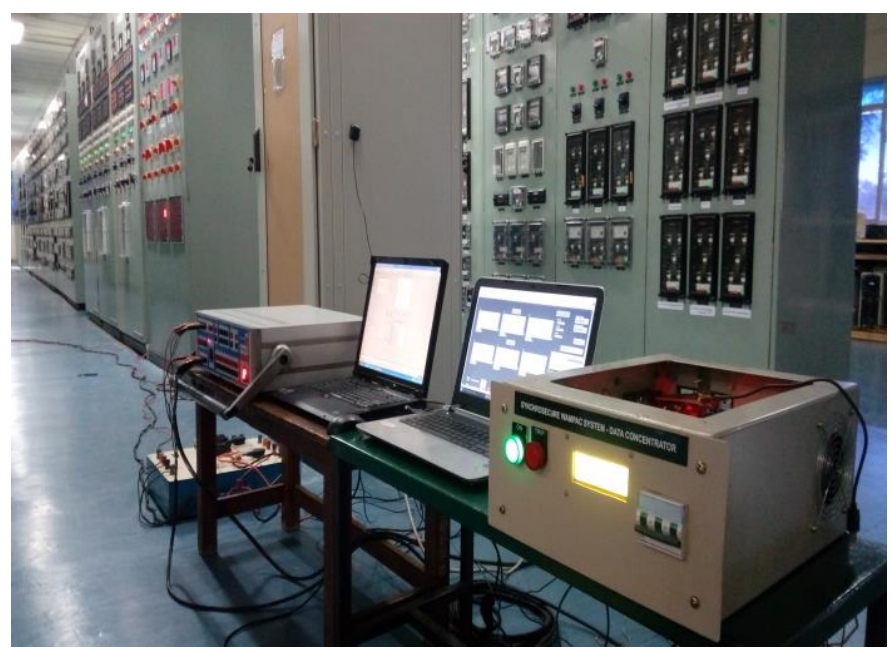

Fig. 11: Real Time Testing of the Proposed Module.

\section{Conclusion}

This Paper has proposed an innovative digital alternative module of Wide Area Monitoring Protection and Control (WAMPAC) System for Power Network. This Digital Version proves to be an effective alternative module to the other existing WAMPAC systems as it out-smarts even minute faults triggered as a result of Unhealthy System Conditions.

Taking the Cost factor into consideration, this module has costed INR 25000 to be handmade, which includes the component cost and fabrication costs. If this module is industry-made in bulk quantities, the cost can be further brought down. The Cost factor makes this module a boon to the entire power sector as the existing WAMPAC modules are priced so high that it extends to around INR 50,00,000 in Indian Rupees. The feasibility and reliability of this module makes it an effective technological advancement at cheaper cost, thereby empowering growing economies to attain better Power System Security.

\section{Acknowledgements}

I express my sincere gratitude to Tamil Nadu Electricity Board (TNEB) for granting me permission to test the designed module at 400/230/110 kV Substation at Alamathy and accrediting it.

\section{References}

[1] A. Carta, N. Locci, and S. Sulis, "A Flexible GPS-Based System for Synchronized Phasor Measurement in Electrical Distribution Networks," IEEE Transactions on Instrumentation and Measurement, vol. 57, no. 11, pp. 2450-2456, 2008.

[2] V. Salehi, A. Mazloomzadeh, and O. Mohammed, "Development and Implementation of a Phasor Measurement Unit for Real-Time Monitoring," Control and Protection of Power Systems, pp. 1 -7, 2011. 